\title{
Checkpoint Inhibition Causing Complete Remission of Metastatic Combined Hepatocellular-Cholangiocarcinoma after Hepatic Resection
}

\author{
Magnus Rizell ${ }^{a} \quad$ Fredrik Åberg $^{a} \quad$ Mats Perman $^{b} \quad$ Lars Ny $^{b}$ \\ Liselotte Stén $^{c}$ Farida Hashimid ${ }^{d}$ Joar Svanvik ${ }^{a}$ Per Lindnér ${ }^{a}$ \\ aTransplant Institute, Sahlgrenska University Hospital, Gothenburg, Sweden; \\ bDepartment of Oncology, Sahlgrenska University Hospital, Gothenburg, Sweden; \\ 'Department of Pathology, Sahlgrenska University Hospital, Gothenburg, Sweden; \\ dDepartment of Radiology, Sahlgrenska University Hospital, Gothenburg, Sweden
}

\section{Keywords}

Checkpoint inhibition · Combined hepatocellular-cholangiocarcinoma $\cdot$ Sorafenib $\cdot$ Immunerelated hepatitis

\begin{abstract}
Combined hepatocellular-cholangiocarcinoma $(\mathrm{CHC})$ is a rare type of primary liver cancer, speculated to arise from hepatic progenitor cells, and with a worse prognosis than hepatocellular carcinoma (HCC). Serum alpha-fetoprotein (AFP) levels may be one prognostic factor. It has been suggested that checkpoint inhibition might be useful in the treatment of HCC where there is an increased expression of PD-1 and PD-L1 in the microenvironment. Its effect on CHC is unknown. We report a case with a large $\mathrm{CHC}$, which was radically resected, but the 53-yearold female patient subsequently developed pulmonary metastases. Histology demonstrated low-differentiated $\mathrm{CHC}$ without microsatellite instability. Treatment with sorafenib was started but was stopped due to angioedema. Under subsequent gemcitabine/cisplatin treatment, the metastatic disease progressed with rising AFP levels. A third-line treatment with pembrolizumab was then started, $2 \mathrm{mg} / \mathrm{kg}$ b.w. i.v. every third week for 6 months. This resulted in a radiologically complete remission of the pulmonary metastases and AFP levels were normalized $(<10 \mu \mathrm{g} / \mathrm{L})$ from a level of $1,790 \mu \mathrm{g} / \mathrm{L}$ before treatment. The patient developed immunerelated adverse events (AEs) including diarrhea and hepatitis. These AEs were successfully treated with prednisolone and mycophenolate mofetil, and they were eventually resolved. There are no signs of cancer recurrence neither in the liver nor in the lungs at 33 months after the start of the checkpoint inhibition treatment, and the patient is doing well. Further study is urgently needed on the role of checkpoint inhibition therapy in liver cancer.
\end{abstract}




\section{Introduction}

Primary liver cancer, chiefly hepatocellular carcinoma (HCC) and cholangiocellular carcinoma (CCC), is a leading malignancy in the world. Despite active treatments, prognosis is often poor. Combined hepatocellular-cholangiocarcinoma ( $\mathrm{CHC}$ ) accounts for $0.4-14 \%$ of primary liver cancers [1]. From the results of the expression of hepatocellular, cholangiocellular and progenitor cell markers, it is speculated that $\mathrm{CHC}$ originates from hepatic progenitor cells [1]. Genetic analyses have shown that CHC is more closely related to CCC than to HCC when it comes to patterns of loss of heterozygosity and beta-catenin and P53 mutations [2]. The prognosis of CHC is worse than for HCC, but better than for CCC [1]. The level of serum alpha-fetoprotein (AFP) may be one prognostic factor [1].

Programmed cell death 1 (PD-1) is a checkpoint molecule expressed by cytotoxic T lymphocytes that is crucial for self-tolerance. Tumor cells can express its ligands, PD-L1 and PD-L2, which by binding to PD-1 leads to T-cell exhaustion with subsequent impairment of immune responses directed against the tumor. This leads to immune tolerance against the cancer. Blocking this binding of tumor cell PD-L1 to PD-1 with a checkpoint inhibitor such as anti-PD-L1 or anti-PD-1 antibodies allows the immune system to destroy cancer cells. Checkpoint inhibitors have been shown to be useful in treating, for example, malignant melanoma, lung cancer and renal cell cancer and are presently being tested for many other malignancies.

Pembrolizumab is a humanized IgG4 isotype antibody that targets the PD-1 receptor of T-lymphocytes. The FDA initially approved pembrolizumab for the treatment of metastatic melanoma. In 2017, the FDA approved it for any unresectable or metastatic solid tumor with certain genetic anomalies such as mismatch repair deficiency or microsatellite instability (MSI).

It has been suggested that checkpoint inhibition could be useful in the treatment of HCC where there is expression of PD-1 and PD-L1 in the tumor microenvironment [3]. It is also suggested that high levels of PD-1 and PD-L1 expression may be useful as biomarkers to predict prognosis in patients with HCC [3]. Finkelmeier et al. [4] demonstrated that high soluble PD-L1 in serum indicated a poor outcome in patients with HCC. In September 2017, the FDA approved another PD-1 blocker, nivolumab, as a second-line treatment for HCC after failure to treatment with sorafenib.

So far there are no reports in the literature on the use of checkpoint inhibitors in CHC. We present a case where surgical resection of a large CHC in the liver and treatment of metachronous pulmonary metastases with pembrolizumab resulted in a complete cancer remission.

\section{Case Report}

A 53-year-old woman presented with a palpable liver tumor confirmed by radiology (Fig. 1). The initial radiology workup showed no signs of extrahepatic tumors. AFP was $167,000 \mu \mathrm{g} / \mathrm{L}$ (reference $<10 \mu \mathrm{g} / \mathrm{L}$ ) and CA19-9 $48 \mathrm{kU} / \mathrm{L}$ (reference $<30 \mathrm{kU} / \mathrm{L}$ ). A radical extended resection of the left lobe and segments $\mathrm{V}$ and VII was performed in February 2016. The resected tumor included a major lesion with the largest diameter of $27 \mathrm{~cm}$ and two satellite lesions sized 25 and $30 \mathrm{~mm}$, respectively. The resection surface was free of cancer.

Histology demonstrated a poorly differentiated CHC. There were no tumor areas with ordinary hepatocytes or cholangiocytes, rather all cells had mixed features of both HCC with trabecular growth and CCC with tendency for glandular formation. There was also a vascular invasion. Lymph nodes (two) were free of cancer. 
Fig. 1. A radical extended resection of the left lobe and segments $\mathrm{V}$ and VII was performed in February 2016. The resected tumor included a major lesion with the largest diameter of $27 \mathrm{~cm}$ and two satellite lesions sized 25 and 30 $\mathrm{mm}$, respectively. The resection surface was free of cancer.

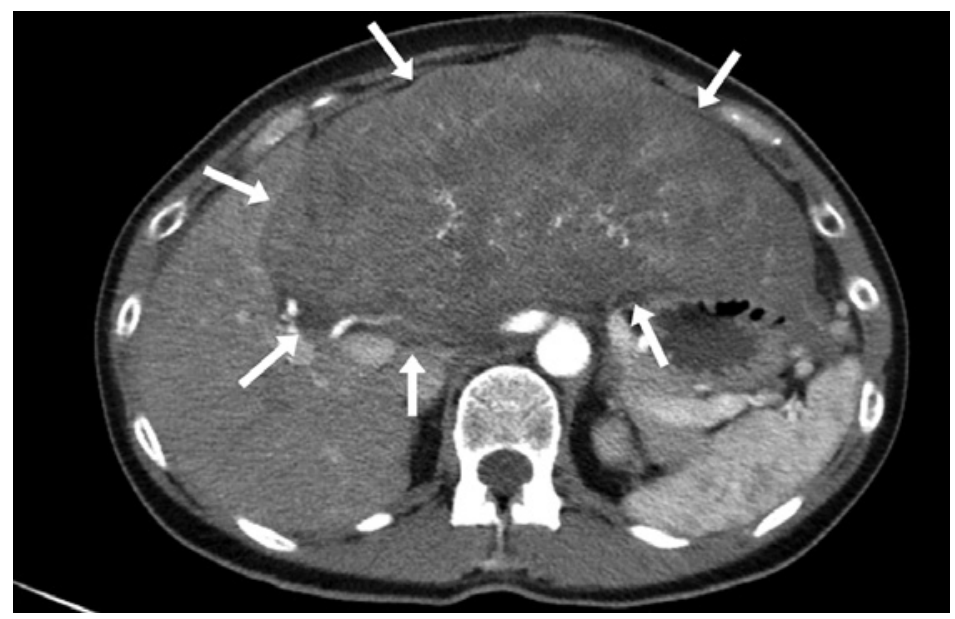

Immunohistochemical staining of the primary liver tumor showed positive responses for Arginase 1, AFP and Glypican 3, Cytokeratin 7 and 19, MUC1, and carbonic anhydrase, and Ki-67 in 20\%. Negative responses were found for hepatocyte, CK20, vimentin, MUC2, SMAD4, WT1, TTF1, CDX2, chromogranin A, synaptophysin, ER, PR, GATA3, KIT, CD10, CD30, CD31, CD34, inhibin and HCG, OCT3/4, TFE3.

Immunohistochemistry was repeated for analysis of MSI in the primary tumor, and this turned out negative, which indicates a normal expression of MMR-proteins; thus, there was no support for MSI.

The patient recovered well from the operation, but at 6 months after surgery, a follow-up computerized tomography (CT) showed 6 metastases in the lungs, with the diameter of the largest nodule being $11 \mathrm{~mm}$. Pulmonary biopsy confirmed metastatic low-differentiated CHC. The pulmonary metastatic tissue was studied for MSI with PCR, but no such signs were found. Immunohistochemical staining demonstrated positivity in CD3, CD4, CD8, and abundant infiltration of lymphocytes, and thus many CD8-positive cytotoxic T-lymphocytes.

PD-L1 analysis using antibody PD-L1 IHC 22C3 pharmDx, Dako/Agilent was performed twice in both the primary tumor and the pulmonary metastases and turned out to be negative.

Sorafenib treatment $(400 \mathrm{mg} \times 2$ ) was started in November 2016, but caused angioedema and was therefore stopped after 1 week. Treatment with gemcitabine and cisplatin, 1,400 mg $\left(1,000 \mathrm{mg} / \mathrm{m}^{2}\right)$ and $35 \mathrm{mg}\left(25 \mathrm{mg} / \mathrm{m}^{2}\right)$, respectively on day 1 and day 8 , and a new cycle on day 29, was started in January 2017. During this treatment, however, there was a doubling of AFP from 710 to $1,590 \mu \mathrm{g} / \mathrm{L}$ and the treatment was therefore stopped after 2 treatment cycles in late February 2017.

A treatment program was then planned with pembrolizumab $2 \mathrm{mg} / \mathrm{kg}$ b.w. i.v. every third week and was started in March 2017. The first response evaluation by CT after 3 months of treatment showed that the number of pulmonary metastases had reduced from 6 to 3 , and the largest diameter had reduced from 11 to $8 \mathrm{~mm}$. The AFP level had dropped from 1790 to $52 \mu \mathrm{g} / \mathrm{L}$ within 2 months and became normal $(<10 \mu \mathrm{g} / \mathrm{L})$ within 4 months after the start of pembrolizumab (Fig. 2).

The patient had some eye problems with blurring of the sight on the right eye. An ophthalmologist diagnosed a vitreous detachment. This was interpreted as being caused by nearsightedness due to mechanical factors and the ophthalmologist could not see a relation with the medication. 


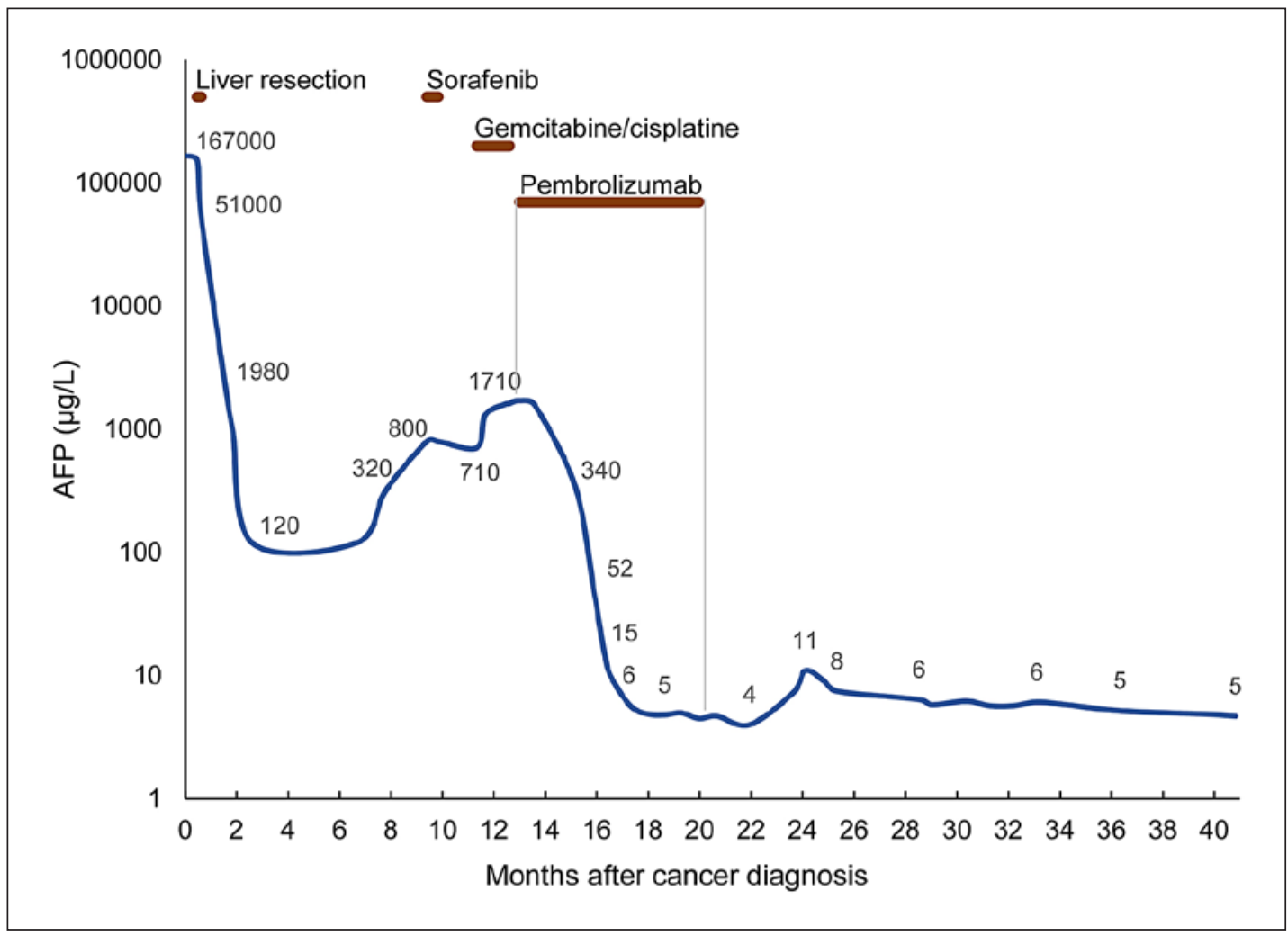

Fig. 2. Serum AFP levels during the course of cancer treatments.

At 6 months following the start of the pembrolizumab treatment, a CT showed reduced sizes of the pulmonary metastases and AFP was stable at $5 \mu \mathrm{g} / \mathrm{L}$. After 9 months of therapy, there was a complete radiological remission of the pulmonary metastases.

After 6 months of pembrolizumab therapy, however, there was a rise in her liver enzymes, with alanine aminotransferase (ALT) up to $940 \mathrm{U} / \mathrm{L}$, aspartate aminotransferase (AST) 765 $\mathrm{U} / \mathrm{L}$, and alkaline phosphatase $330 \mathrm{U} / \mathrm{L}$, but normal plasma bilirubin and INR. This led to the cessation of pembrolizumab treatment in October 2017. A liver biopsy demonstrated mild portal and lobular inflammation, compatible with hepatitis with inflammatory grade 1-2/4. The inflammatory infiltration contained mainly lymphocytes, but also neutrophils, eosinophils and ceroid-containing macrophages. Fibrosis stage varied from 0 to $2 / 4$ according to Batts and Ludwig.

Antinuclear antibodies, smooth muscle antibodies, liver-kidney-microsomal antigen-1 antibodies, and anti-mitochondrial antibodies were all negative. Tests for viral hepatitis A, B, $\mathrm{C}$ and $\mathrm{E}$, as well as cytomegalovirus were negative. Serum immunoglobulin G level was 1.3 $\mathrm{g} / \mathrm{L}$ (reference 6.7-15.0 g/L). The findings were compatible with checkpoint inhibitorinduced immune-related hepatitis.

The patient was treated with prednisolone, initially $80 \mathrm{mg}$, then $40 \mathrm{mg}$, with subsequent dose reductions by 5-10 mg. Liver enzymes responded well to high-dose prednisolone, but once prednisolone doses were reduced to $25-30 \mathrm{mg} /$ day, there were repeated relapses with rising ALT and AST. Due to the dependency on high-dose steroids, it was decided to start treatment with mycophenolate mofetil (MMF) in January 2018, initially 500 mg twice daily, and then raised to $1 \mathrm{~g}$ twice daily. By this time, around 9 months after the start of pembrolizumab, the patient had also developed nonhemorrhagic diarrhea and leg edema with hypo- 
albuminemia $(19 \mathrm{~g} / \mathrm{L})$. All of these side effects, however, resolved by 2 weeks after initiation of MMF treatment. The liver enzymes became normal and have remained normal during follow-up. Prednisolone was slowly tapered, and stopped in September 2018, 11 months after her presentation with hepatitis. MMF was stopped in April 2019. As by January 2020, 33 months after the start of the checkpoint inhibition treatment, the patient is doing well, and AFP remains normal. Liver enzymes are all within reference limits.

The most recent CT of the thorax and abdomen from September 2019 (30 months after the start of pembrolizumab) shows no signs of malignancy.

\section{Methods}

\section{Immunohistochemistry}

The mismatch repair proteins MLHI, PMS2, MSH2 and MSH6 in tumor tissue were analyzed using immunohistochemistry. Formalin-fixed tumor sections were analyzed for MLHI according to instructions from Dako (antibody: monoclonal mouse anti-human Mutl protein homolog 1, code M3640), PMS2 according to instructions from Epitomics (using rabbit monoclonal anti-human PMS2, clone EP51) [5], MSH2, according to instructions using monoclonal antibody OAI 219 T60 and MSH6 according to instructions from Epitomics (rabbit monoclonal antibody EP49, rabbit IgG) as previously described [6].

PD-L1 analysis was performed with immunohistochemistry according to instructions from Dako (antibody PD-L1 IHC 22C3 pharmDx, Dako/Agilent).

\section{Evaluation of Tumor MSI with Immunochemistry}

Immunohistochemical analyses for: arginase 1, AFP and glypican 3, cytokeratin 7 and 19, MUC 1, and carbanhydrase, Ki-67, CK20, vimentin, MUC2, SMAD4, WT1, TTF1, CDX2, chromogranin A, synaptophysin, ER, PR, GATA3, KIT, CD10, CD30, CD31, CD34, inhibin and HCG, OCT3/4, and TFE3 were performed according to regular clinical praxis.

\section{Evaluation of Tumor MSI with PCR}

The evaluation was done using 5 quasimonomorphic mononucleotide repeats and pentaplex PCR according to Suraweera et al. [7].

\section{Discussion}

The prognosis of CHC is in general better than that for CCC, but worse than that for HCC. The overall 3- and 5-year survival in CHC is reported to be 30 and $18 \%$, respectively [1]. The prognosis in metastasized CHC is even poorer.

The large CHC was poorly differentiated and with vascular invasion but tests for viral hepatitis A, B, C and E, as well as cytomegalovirus were negative. There were several poor prognostic factors in the patient. She had a large tumor, initially very high AFP levels, later cisplatin rising AFP during gemcitabine-cisplatin therapy. Large tumor diameter, high AFP and CA19-9 and vascular invasion are all poor prognostic signs [8]. Subsequent pembrolizumab therapy, however, led to a rapid and dramatic treatment response with complete remission that has now been maintained for 33 months from the start of pembrolizumab therapy.

CHC is commonly divided into a classical type and a rarer type with stem cell features. CHC should be separated from the rare condition of having two different tumors (HCC and CCC) simultaneously. The tumor in the present case was a classical type of CHC with the presence of cells with features of both CCC and HCC. 
AFP was initially high, $167,000 \mu \mathrm{g} / \mathrm{L}$, before the liver resection, while CA19-9 was only moderately elevated. In a series of 45 patients with $\mathrm{CHC}, 62 \%$ had an elevated AFP, while only $22 \%$ had an elevated CA19-9 [9].

Immune checkpoint inhibitors act by blocking the T-cell checkpoint receptors, such as PD-1, that are responsible for T-cell exhaustion, and thereby potentiate a strong immune response directed against the tumor. Tumors with deficits in DNA mismatch repair can lead to MSI with the accumulation of thousands of somatic mutations. This high mutational burden makes such tumors immunogenic and thereby sensitive to immune checkpoint inhibitor therapies [10]. Indeed, the degree of MSI and mutational load seems to influence the response rate to checkpoint inhibition therapy in general. Interestingly, although we were unable to demonstrate MSI in the tumor, there was still a dramatic response to pembrolizumab on the lung metastases.

By unbalancing the immune system, immune checkpoint inhibitor therapies can trigger autoimmune-like adverse effects, of which gastrointestinal manifestations are fairly common [11]. Immune-related hepatitis affects an estimated $1-4 \%$ of patients treated with anti-PD-1 antibodies, and 4-9\% of those treated with anti-CTLA-4 antibodies [11]. The patient suffered from both diarrhea and immune-related hepatitis, as well as leg edema that might have been secondary to hypoalbuminemia. These side effects led to cessation of pembrolizumab therapy but resolved with immunosuppressive therapy. Despite immunosuppression therapy, as to date, there are no signs of tumor progression in this case.

Accumulating experience support the use of corticosteroids as first-line treatment of severe immune-related side effects and immunosuppression with, for instance, MMF as second-line $[12,13]$. Adverse effects and their treatments do not seem to impair the effects of checkpoint inhibitors.

In conclusion, given the dramatic and long-lasting response to checkpoint inhibition therapy in this case with advanced metastatic CHC, further trials are urgently needed in this type of primary liver cancer.

\section{Statement of Ethics}

This case report was conducted in accordance with the Declaration of Helsinki. Written informed consent was obtained from the patient for publication of this article. As this is a retrospective description of a case treated according to clinical practice, no approval from the ethics committee was considered necessary.

\section{Disclosure Statement}

The authors have no conflicts of interest to declare.

\section{Funding Sources}

None received. 
Rizell et al.: Checkpoint Inhibition in Combined Hepatocellular-Cholangiocarcinoma

\section{Author Contributions}

M.R., F.A., and P.L. are responsible for the surgical care and M.P. and L.N. for the oncologic care of the patient. F.A. and J.S. for conception of the study and drafting the manuscript. L.S. is responsible for the pathology examination of the primary liver tumor and pulmonary metastases including histology, immunohistochemistry analyses of MSI. F.H. is responsible for the radiological examinations of the patient. All authors were involved in writing the final manuscript.

\section{References}

1 Wang AQ, Zheng YC, Du J, Zhu CP, Huang HC, Wang SS, et al. Combined hepatocellular cholangiocarcinoma: controversies to be addressed. World J Gastroenterol. 2016 May 14;22(18):4459-65.

2 Cazals-Hatem D, Rebouissou S, Bioulac-Sage P, Bluteau O, Blanché H, Franco D, et al. Clinical and molecular analysis of combined hepatocellular-cholangiocarcinomas. J Hepatol. 2004 Aug;41(2):292-8.

3 Moris D, Rahnemai-Azar AA, Zhang X, Ntanasis-Stathopoulos I, Tsilimigras DI, Chakedis J, et al. Program death-1 immune checkpoint and tumor microenvironment in malignant liver tumors. Surg Oncol. 2017 Dec; 26(4):423-30.

4 Finkelmeier F, Canli Ö, Tal A, Pleli T, Trojan J, Schmidt M, et al. High levels of the soluble programmed deathligand (sPD-L1) identify hepatocellular carcinoma patients with a poor prognosis. Eur J Cancer. 2016 May;59: 152-9.

5 Gark K, Soslow RA. Lynch syndrome (hereditary non-polyposis colorectal cancer) and endometrial carcinoma. J Clin Pathol. 2009 Aug;62(8):679-84.

6 Shia J, Tang LH, Vakiani E, Guillem JG, Stadler ZK, Soslow RA, et al. Immunohistochemistry as first-line screening for detecting colorectal cancer patients at risk for hereditary nonpolyposis colorectal cancer syndrome: a 2-antibody panel may be as predictive as a 4-antibody panel. Am J Surg Pathol. 2009 Nov;33(11):1639-45

7 Suraweera N, Duval A, Reperant M, Vaury C, Furlan D, Leroy K, et al. Evaluation of tumor microsatellite instability using five quasimonomorphic mononucleotide repeats and pentaplex PCR. Gastroenterology. 2002 Dec; 123(6):1804-11.

8 Tian MX, Luo LP, Liu WR, Deng W, Yin JC, Jin L, et al. Development and validation of a prognostic score predicting recurrence in resected combined hepatocellular cholangiocarcinoma. Cancer Manag Res. 2019 Jun 5;11:5187-95.

9 Li R, Yang D, Tang CL, Cai P, Ma KS, Ding SY, et al. Combined hepatocellular carcinoma and cholangiocarcinoma (biphenotypic) tumors: clinical characteristics, imaging features of contrast-enhanced ultrasound and computed tomography. BMC Cancer. 2016 Feb 25;16:158.

10 Mandal R, Samstein RM, Lee KW, Havel JJ, Wang H, Krishna C, et al. Genetic diversity of tumors with mismatch repair deficiency influences anti-PD-1 immunotherapy response. Science. 2019 May 3;364(6439):485-91.

11 Nishida N, Kudo M. Liver damage related to immune checkpoint inhibitors. Hepatol Int. 2019 May;13(3): 248-52.

12 Brahmer JR, Lacchetti C, Thompson JA, Atkins MB, Brassil KJ, Caterino JM, et al. Management of immunerelated adverse events in patients treated with immune checkpoint inhibitor therapy: American society of clinical oncology clinical practice guideline summary. J Oncol Pract. 2018 Jun 10;14 (4):247-9.

13 De Martin E, Michot JM, Papouin B, Champiat S, Mateus C, Lambotte O, et al. Characterization of liver injury induced by cancer immunotherapy using immune checkpoint inhibitors. J Hepatol. 2018 Jun;68(6):1181-90. 